\title{
Problema de Localización y Ruteo con Restricciones de Capacidad: Revisión de la Literatura
}

\author{
The capacitated location routing problem: review of literature
}

Problema de localização e roteamento com restrições de
capacidade: revisão da literatura

Fecha de Recepción: 06 de Febrero de 2015

Fecha de Aceptación: 18 de Abril de 2015

\author{
John Willmer Escobar* \\ Rodrigo Linfati** \\ Wilson Adarme Jaimes ${ }^{\star \star *}$
}

\section{Resumen}

En este artículo se hace una revisión exhaustiva del estado del arte de las metodologías de solución existentes para el problema combinado de localización y ruteo con restricciones de capacidad (CLRP). El problema de CLRP tiene una gran cantidad de aplicaciones prácticas en temas relacionados con transporte. Se ha propuesto el siguiente esquema de clasificación de acuerdo al método de solución: (1) Algoritmos Heurísticos Constructivos, (2) Algoritmos Heurísticos Basados en Clústeres, (3) Algoritmos Heurísticos Basados en Trayectoria, (4) Algoritmos Heurísticos Basados en Población, (5) Algoritmos Heurísticos Combinados, (6) Métodos Exactos. Se hace especial énfasis en fortalezas y debilidades de cada metodología publicada, identificando oportunidades de investigación y desarrollo en el área, en el contexto de la aplicación práctica de la problemática.

Palabras claves: Problemas de localización y ruteo, Revisión Literatura, Algoritmos Metaheurísticos, Métodos Exactos.

\section{Abstract}

In this paper, we review the state of the art of the published solution methods for combined problems of location and routing with capacity constraints (CLRP). The CLRP has several practical application in topics related to transportation. We have proposed the following classification scheme based on the solution method: (1) Constructive Heuristics Algorithms, (2) Heuristic Algorithms Based on Clusters, (3) Heuristic Algorithms Based on Trajectory,

* Ph.D. Pontificia Universidad Javeriana Cali (Cali - Valle del Cauca, Colombia). jwescobar@javerianacali.edu.co.

** Ph.D. Universidad del Bío-Bío (Concepción, Chile). rlinfati@ubiobio.cl.

*** Ph.D. Universidad Nacional de Colombia (Bogotá - Distrito Capital, Colombia).wadarmej@unal.edu.co. 
(4) Heuristic Algorithms Based on Population, (5) Combined Heuristic Algorithms, (6) Exact Methods. Special emphasis is placed on the fortress and on the lack of each published method, identifying research opportunities in the context of the real application of the problem.

Keywords: Location Routing Problem, Literature Review, Metaheuristic Algorithms, Exact Methods.

\section{Resumo}

Apresenta uma revisão exaustiva do estado da arte das metodologias de solução existentes para o problema combinado de localização e roteamento com restrições de capacidade (CLRP). O CLRP tem grande quantidade de aplicações práticas em temas relacionados com transporte. Tem-se proposto o seguinte esquema de classificação, de acordo com o método de solução: (1) Algoritmos heurísticos construtivos, (2) Algoritmos heurísticos baseados em clusters, (3) Algoritmos heurísticos baseados em trajetória, (4) Algoritmos heurísticos baseados em população, (5) Algoritmos heurísticos combinados, (6) Métodos exatos. Faz-se especial ênfase nos pontos fortes e fracos de cada metodologia publicada, identificando oportunidades de pesquisa e desenvolvimento na área, no contexto da aplicação prática da problemática.

Palavras chave: Problemas de localização e roteamento, Algoritmos Meta-heurísticos, Métodos Exatos.

Cómo citar este artículo:

[1] J.W. Escobar, R. Linfati \& W.A. Jaimes, "Problema de Localización y Ruteo con Restricciones de Capacidad: Revisión de la Literatura”, Fac. Ing., vol. 24 (39), pp. 85-98, Mayo-Ago. 2015. 


\section{INTRODUCCIÓN}

El estudio de problemas combinados de logística es un área de investigación relativamente nueva [1]. Una de las problemáticas comunes en este campo es la consideración de decisiones de localización y ruteo simultáneamente. Los diferentes aspectos de estas decisiones tales como la localización, asignación y ruteo han sido estudiados generalmente de manera independiente. Este hecho puede ser explicado debido a que la localización es una decisión estratégica la cual es tomada por un largo período de tiempo, mientras que el ruteo es una decisión operacional que puede ser modificada dinámicamente muchas veces en un período corto de tiempo. Sin embargo estas decisiones están íntimamente relacionadas. De hecho, la decisión de localización de un depósito es frecuentemente influenciada por los costos de transporte y viceversa [2].

Este artículo presenta una revisión exhaustiva de los métodos de solución para el problema de localización y ruteo con restricciones de capacidad (CLRP). El CLRP puede ser modelado mediante el siguiente problema de grafos: Sea un grafo no dirigido indirecto, donde es un conjunto de nodos que contiene un subconjunto, de depósitos potenciales y un subconjunto de clientes. Cada depósito potencial tiene una capacidad y un costo de apertura. Cada cliente tiene una demanda, la cual debe ser satisfecha por un depósito abierto. Un conjunto de vehículos idénticos, cada uno con capacidad y costo fijo, está disponible en cada depósito. Cada arco tiene asociado un costo de viaje que es proporcional a la distancia entre ambos puntos.

El objetivo del CLRP es determinar los depósitos a ser abiertos, los clientes a ser asignados a cada depósito abierto y las rutas a ser desarrolladas para satisfacer las demandas de los clientes. Una solución factible para el CLRP debe considerar las siguientes restricciones: i) cada ruta debe comenzar y finalizar en el mismo depósito; ii) cada cliente debe ser visitado exactamente una vez en una sola ruta; iii) la carga total de cada ruta no debe exceder la capacidad del vehículo; iv) La carga total de las rutas asignadas a un depósito abierto no debe exceder su capacidad.

La función objetivo del CLRP está determinada por la suma de los costos de apertura, de los costos de viaje y de los costos fijos asociados con los vehículos usados. El CLRP es conocido por ser un problema NP-hard, dado que este es una generalización de dos problemas NP-hard: el problema de localización de instalaciones con restricciones de capacidad (CFLP) y el problema de ruteo de vehículos con múltiples depósitos (MDVRP).

En la sección 1 se describe la literatura relevante asociada al problema CLRP, clasificada en 6 categorías, de acuerdo a la metodología de solución empleada. En la sección 2 se presentan las conclusiones y las oportunidades de investigación futuras. En algunas secciones se muestra una tabla que resume los resultados obtenidos por los algoritmos desarrollados para el CLRP sobre el set de instancias propuesto para la problemática. Se ha realizado un análisis de la calidad de las soluciones y el tiempo computacional obtenido por cada uno de los diferentes métodos de solución. Se espera que este artículo pueda dar una orientación general sobre posibilidades de investigación en el área para estudiantes de doctorado, estudiantes de maestría y profesores que trabajan en el área logística.

\section{REVISIÓN DE LA LITERATURA}

Para el problema CLRP se han propuesto tres set de instancias de benchmarking en la literatura. El primer set de datos propuesto (Tuzun Instances) en [3] considera 36 instancias. El número de clientes esta determinado en el intervalo $(100,200)$, y el número de depósitos potenciales es 10 o 20. La capacidad del vehículo es determinada como 150. El segundo set de instancias (Prodhon Instances) es introducido en [4] y considera 30 instancias con restricciones de capacidad en depósitos y vehículos. El número de depósitos potenciales es 5 o 10 y el número de clientes es 20, 50, 100 o 200. Los clientes y los depósitos corresponden a puntos aleatorios en el plano cartesiano. En este set de instancias, el costo de viaje es calculado como la distancia euclidiana de una ruta multiplicada por 100 y redondeado al entero más cercano. La capacidad del vehículo es 70 o 150 y las demandas de los clientes son uniformemente distribuidas entre el intervalo $(11,20)$. Finalmente, el último set de datos (Barreto Instances) propuesto en [5] considera 19 instancias obtenidas de problemas clásicos de ruteo de vehículos con restricciones de capacidad adicionando nuevos depósitos con sus correspondientes capacidades y 
costos fijos. El número de clientes varía entre 21 y 150 , y el número de depósitos potenciales de 5 a 10.

Pocos artículos se han dedicado a la revisión de la literatura para problemas combinados de localización y ruteo (LRP) [6-9]. A pesar de que estos trabajos se enfocan de manera general en la revisión del estado del arte para el LRP, se ha recopilado alguna información relevante sobre el problema CLRP, el cual es una variante del LRP considerando restricciones de capacidad en depósitos y rutas. En [6], se presenta una revisión de la literatura para el problema de localización y ruteo (LRP) basados en dos aspectos: perspectiva del problema y método de solución. Dentro de la perspectiva del problema, se consideran 11 elementos dentro de los cuales se destacan la naturaleza de la demanda (determinística o estocástica), número de instalaciones (una o múltiples), tamaño de la flota de vehículos (un vehículo o múltiples vehículos), horizonte de planeación (un período o múltiples períodos), entre otros. Respecto al método de solución, dos categorías son consideradas: algoritmos exactos y algoritmos heurísticos. En [7], se presenta una clasificación más específica de los problemas de LRP basada en 9 aspectos, dentro de los cuales se destacan el tipo de función objetivo, el número y tipo de vehículos, el método de solución, la estructura de las rutas, entre otras. Recientemente, en [8] se muestra una corta descripción de la problemática relacionada con el LRP, algunas aplicaciones prácticas y algunas sugerencias futuras de investigación; mientras que en [9], se hace una recopilación reciente de los algoritmos más efectivos mostrando las oportunidades de investigación para todas las variantes del LRP.

En este paper, nosotros consideramos la clasificación de los algoritmos propuestos de solución para el CLRP de acuerdo a la metodología de solución empleada, de la siguiente manera: (1) Algoritmos Heurísticos Constructivos, (2) Algoritmos Heurísticos Basados en Clústeres, (3) Algoritmos Heurísticos Basados en Trayectoria, (4) Algoritmos Heurísticos Basados en Población, (5) Métodos Exactos y (6) Algoritmos Heurísticos Combinados. En cada sección se detallan algunos aspectos relacionados con cada algoritmo propuesto.

\section{A. Algoritmos Heurísticos Constructivos}

Los algoritmos heurísticos constructivos se orientan a la generación de una solución inicial a partir del análisis y selección de los componentes que la conforman. Dos trabajos relevantes en esta categoría para la solución del CLRP, son los publicados por [4] y [10]. En [4], se presenta un algoritmo GRASP con una estrategia de post optimización basada en Path-relinking para resolver el CLRP. Una versión extendida del algoritmo de los ahorros propuesto en [11] es utilizado como la heurística aleatoria usada por el GRASP. La principal fortaleza de este algoritmo es que permite la construcción de una solución inicial en un tiempo de cómputo reducido, lo que permite aplicar procedimientos de mejoramiento a posteriori. Como en el CLRP los resultados son sensitivos al costo de los depósitos, este algoritmo ha demostrado encontrar buenas soluciones para instancias donde los depósitos no tienen restricciones de capacidad.

En [10], se usa el procedimiento GRASP descrito en [4] para crear una solución inicial, la cual es mejorada a través de una búsqueda local evolutiva (ELS). Una de las principales fortalezas de dicho algoritmo es que utiliza las dos potencialidades del GRASP para construir una solución inicial en tiempo reducido y la eficiencia de la ELS propuesta en [12]. El algoritmo propuesto es ejecutado cinco veces y solamente los mejores resultados son reportados con el tiempo computacional requerido para alcanzarlos. Esto hace que la comparación de resultados obtenidos por este método con otros publicados pueda ser errónea. En la Tabla 1 se muestran los resultados obtenidos de los algoritmos GRASP [4] y GRASP+ELS [10] sobre los sets de instancias de benchmarking. 
TABla 1.

RESULTADOS OBTENIDOS PARA ALGORITMOS GRASP y GRASP+ELS

\begin{tabular}{|c|c|c|c|c|c|c|c|}
\hline & \multicolumn{2}{|c|}{ Tuzun Instances } & \multicolumn{2}{|c|}{ Prodhon Instances } & \multicolumn{2}{|c|}{ Barreto Instances } & \multirow{2}{*}{ CPU } \\
\hline & Gap BKS & CPU time & Gap BKS & CPU time & Gap BKS & CPU time & \\
\hline GRASP [4] & 3.50 & 163 & 3.63 & 97 & 1.63 & 20 & $\begin{array}{l}\text { Intel Pentium } 4 \\
(2.40 \mathrm{Ghz})\end{array}$ \\
\hline GRASP+ELS [10] & 1.29 & 607 & 1.09 & 258 & 0.08 & 188 & $\begin{array}{c}\text { Intel Core 2 Quad } \\
\quad(2.83 \mathrm{Ghz})\end{array}$ \\
\hline
\end{tabular}

Fuente: Realizada por los autores

La notación utilizada en Tabla 1 es la misma utilizada en las tabas subsiguientes en el artículo. La columna Gap BKS corresponde a la variación porcentual del costo encontrado por cada algoritmo con respecto al valor del mejor resultado reportado en la literatura (BKS). La columna CPU time, indica el tiempo de ejecución en segundos en la CPU usada por cada algoritmo. Finalmente, la última columna (CPU) indica la CPU usada por cada algoritmo.

\section{B. Algoritmos Heurísticos Basados en Clústeres}

Estos métodos generalmente construyen una solución inicial mediante la generación de clústeres de clientes. Luego se procede a asignar dichos clústeres a un depósito potencial, para luego resolver el correspondiente problema de ruteo de vehículos (VRP). Tres trabajos han considerado técnicas basadas en clústeres para el CLRP [5], [13] y [14].

En [5], se proponen diferentes técnicas de clústeres jerárquicas y no jerárquicas, con diferentes funciones de proximidad para agrupar los clientes dentro del contexto del CLRP. En este trabajo, se ha demostrado que la formación de clústeres determina buenas soluciones iniciales para el CLRP, en tiempo de soluciones aceptables; de manera que sirva de apoyo al proceso de toma de decisiones en el ámbito real.

En [13], se utiliza un esquema de solución del CLRP basado en cuatro pasos principales: (i) construcción de grupos de clientes con una capacidad limitada, (ii) determinación de la distribución de los clientes en cada grupo, (iii) mejora de la secuenciación de los clientes mediante la solución de un problema de viajero de negocios (TSP) y (iv) localización de los depósitos y asignación de los grupos de clientes. Todos estos aspectos se han involucrado en una herramienta de soporte a la decisión desarrollada para ambiente Windows.

En [14] se propone una versión heurística de 4 fases para el CLRP. En la primera fase, los clientes son agregados en clústeres utilizando un algoritmo constructivo básico llamado greedy clustering method. En la segunda fase, el centro de gravedad de cada clúster es calculado. Luego en la tercera fase, los depósitos requeridos para cubrir la demanda total de los clientes son localizados, teniendo en cuenta la minimización del costo total de apertura. Los grupos de clientes son asignados a los depósitos abiertos considerando la distancia existente de los centros de gravedad de los clústeres hacia los depósitos. Finalmente, en la cuarta fase, las rutas son mejoradas mediante la aplicación de un algoritmo basado en Colonia de Hormigas (AC). Solamente, resultados computacionales en un solo grupo de instancias son considerados en [5] y [14]. Al parecer, los resultados obtenidos por estos algoritmos no son tan promisorios, razón por la cual no se han reportado resultados disponibles en todas las instancias para el CLRP. De igual manera, dichos algoritmos no han sido comparados con los más efectivos publicados hasta la fecha de publicación de los artículos. Una de las debilidades fundamentales de [14], podría ser la falta de consideración del costo de la distancia recorrida desde cada clúster hacia cada depósito, en la decisión de apertura de los mismos. De igual manera, el algoritmo de mejoramiento propuesto en la última fase no considera el intercambio entre depósitos abiertos y cerrados, lo que podría permitir explorar nuevas partes del espacio de solución. 


\section{Algoritmos Heurísticos Basados en Trayectoria}

Los algoritmos heurísticos basados en trayectoria normalmente parten de una solución inicial construida mediante algún procedimiento heurístico e iterativamente tratan de mejorarla moviéndose al vecindario con mejor calidad. Varios trabajos han sido propuestos utilizando esta metodología [3] y [1521]. En [3], se propone un algoritmo basado en una búsqueda tabú de dos fases: localización y ruteo. En la fase de localización, la búsqueda tabú es focalizada en las variables de localización tratando de determinar los "mejores" depósitos a abrir. Por cada fase de localización, una búsqueda tabú es ejecutada sobre las variables de ruteo, de manera que se pueda obtener una buena solución para dicha configuración.

En [15], se propone un algoritmo de dos fases. En la primera fase, los clientes son agregados en "súper clientes" (clientes agregados en una ruta) resolviendo el correspondiente problema de localización de instalaciones con restricciones de "single sourcing", de manera que cada "súper cliente" sea solamente servido por un solo depósito. En primera instancia, el problema de localización se soluciona mediante una relajación langreana de las restricciones de asignación. En la segunda fase, las rutas del problema resultante (MDVRP) son mejoradas usando una búsqueda tabú granular con un solo vecindario. Una de las grandes ventajas de este algoritmo es que permite obtener "buenas" soluciones en tiempos computacionales reducidos, debido a que se limita el espacio de búsqueda local en el proceso de mejora de las rutas.

En [16], se hace una propuesta interesante de un algoritmo basado en simulado y recocido (SA) para el problema del CLRP. En este trabajo, la solución inicial es obtenida mediante una heurística constructiva que mezcla conceptos de localización y ruteo simultáneamente. Tres vecindarios aleatorios son considerados dentro de la estructura algorítmica del SA. Solamente resultados correspondientes a una sola corrida del algoritmo son presentados, sin utilizar diferentes semillas para la generación de números aleatorios. Esta es una limitante debido a que sería importante conocer el desempeño del algoritmo SA utilizando diversas semillas aleatorias, de manera que se pueda comprobar la eficiencia de los vecindarios seleccionados. A pesar de que los resultados obtenidos son buenos respecto a la mejor solución conocida reportada en esa fecha, el tiempo computacional es elevado debido a que se consideran intercambios de depósitos abiertos y cerrados dentro de los vecindarios; siendo este el movimiento más complejo en la solución del CLRP.

Dos algoritmos basados en búsquedas de vecindario variable (VNS) han sido propuestos en [17] y [18]. En [17] se presenta una búsqueda de vecindario variable secuencial (VND) con 7 vecindarios. La solución inicial es construida mediante una heurística basada en la asignación de los clientes más cercanos a los depósitos y la solución del correspondiente problema de ruteo de vehículos para cada uno de ellos utilizando una variante del algoritmo propuesto en [11]. Se puede notar que los resultados reportados en este trabajo son peores a los presentados en otros trabajos. Una de las problemáticas principales de este algoritmo es la falta de consideración de los costos fijos de apertura de depósitos en la fase de inicialización del algoritmo. Esto determina que dentro de la estructura del VND se deban considerar decisiones de cierre y apertura de depósitos que pueden hacer que el algoritmo caiga fácilmente en un óptimo local. En [18] se utiliza igualmente una estructura de VND con 5 vecindarios. Solamente resultados para 13 instancias son reportados, lo que dificulta la comparación del desempeño con los algoritmos más efectivos propuestos para el CLRP.

En [19] se presenta un algoritmo basado en una búsqueda local adaptativa (ALNS) para la solución del problema ruteo de vehículos con decisiones de localización de dos escalones (2E-VRP). Este algoritmo es ejecutado en las instancias de benchmarking para el CLRP obteniendo excelentes resultados. Nuevas estructuras de vecindades son propuestas teniendo en cuenta la problemática de localización y ruteo. La técnica de ALNS fue introducida exitosamente en [22] para el problema de ruteo de vehículos considerando despachos y recogidas con ventanas de tiempo. Una de las ventajas principales de esta metodología radica en que se asignan puntajes para las vecindades, aumentando la probabilidad de selección a aquellas que permiten obtener mejoras en la solución actual. A pesar de ello, para encontrar buenas soluciones se hace necesario la ejecución de muchas iteraciones del algoritmo lo cual incrementa el tiempo computacional. En [20], se propone un algoritmo basado en recocido simulado (SA) con 3 vecindades para el CLRP. Al igual que en [17], solamente se reportan resultados 
para un pequeño número de instancias. El algoritmo no es comparado con las estrategias de solución más eficientes para el CLRP.

Recientemente en [21] han propuesto un algoritmo eficiente que combina la potencialidad de la búsqueda tabú granular (GTS) con las bondades de la búsqueda de vecindario variable (VNS). En dicho algoritmo VNS controla el cambio de los vecindarios mientras que la GTS reemplaza la búsqueda local tradicional en el
VNS. Excelentes resultados computacionales sobre los tres set de instancias son obtenidos. De hecho, nuevas soluciones no conocidas (BKS) son alcanzadas dentro de tiempos computacionales reducidos.

La Tabla 2 presenta un resumen de los resultados obtenidos por los algoritmos propuestos en esta clasificación sobre las instancias propuestas en literatura.

TABla 2.

RESULTADOS OBTENIDOS PARA ALGORITMOS BASADOS EN TRAYECTORIA

\begin{tabular}{|c|c|c|c|c|c|c|c|}
\hline & \multicolumn{2}{|c|}{ Tuzun Instances } & \multicolumn{2}{|c|}{ Prodhon Instances } & \multicolumn{2}{|c|}{ Barreto Instances } & \multirow{2}{*}{ CPU } \\
\hline & Gap BKS & CPU time & Gap BKS & CPU time & Gap BKS & CPU time & \\
\hline TS [3] & 4.32 & 12 & - & - & - & - & $\begin{array}{l}\text { Intel Pentium } 2 \\
\quad(2.66 \mathrm{Mhz})\end{array}$ \\
\hline LRGTS [15] & 1.84 & 22 & 0.76 & 18 & 1.52 & 54 & $\begin{array}{l}\text { Intel Pentium } 4 \\
\quad(2.40 \mathrm{Ghz})\end{array}$ \\
\hline SALRP [16] & 1.49 & 826 & 0.44 & 422 & 0.29 & 161 & $\begin{array}{c}\text { Intel Core } 2 \\
\text { Quad }(2.66 \mathrm{Ghz})\end{array}$ \\
\hline VND [17] & 5.93 & - & - & - & 5.43 & - & $\begin{array}{c}\text { Intel Core } 2 \text { Duo } \\
\quad(2.00 \mathrm{Ghz})\end{array}$ \\
\hline VNS [18] & - & - & - & - & 0.09 & - & $\begin{array}{l}\text { Intel Core } 2 \text { Duo } \\
\quad(2.40 \mathrm{Ghz})\end{array}$ \\
\hline ALNS [19]* & 0.18 & 8103 & 0.25 & 4221 & 0.05 & 1772 & $\begin{array}{c}\text { AMD Opteron } \\
275(2.20 \mathrm{Ghz})\end{array}$ \\
\hline GVTNS [21] & 0.76 & 201 & 0.35 & 91 & 0.67 & 53 & $\begin{array}{l}\text { Intel Core } 2 \text { Duo } \\
\quad(2.00 \mathrm{Ghz})\end{array}$ \\
\hline
\end{tabular}

* Mejores resultados reportados para 5000 iteraciones Fuente: Realizada por los autores.

\section{Algoritmos Heurísticos Basados en Población}

Las heurísticas basadas en población están enfocadas en procedimientos que consideran conjuntos de soluciones que evolucionan sobre un espacio de búsqueda. Las diferentes heurísticas basadas en población se distinguen por la forma en que la información aportada por individuos (elementos de la población) evoluciona para obtener nuevas soluciones. A diferencia de los algoritmos basados en trayectoria, los algoritmos poblacionales realizan un proceso de búsqueda sobre el espacio de soluciones completo. Dentro de esa categoría varios trabajos han sido propuestos [23-25]. En [23] y [24] se proponen dos algoritmos basados en aproximación memética. En [23] se presenta un algoritmo memético con administración de la población (MA|PM) para resolver el problema CLRP. En particular, pequeñas poblaciones están controladas aceptando nuevas soluciones, si la distancia de la población excede un parámetro determinado. En [24] se propone un algoritmo memético que incluye una heurística para la generación inicial de cromosomas, un esquema basado en una búsqueda eficiente y un procedimiento de crossover. Resultados computacionales para el CLRP muestran la eficiencia de estos algoritmos; sin embargo los tiempos computacionales reportados son altos 
en comparación con algunos algoritmos heurísticos basados en trayectoria.

Un algoritmo basado en múltiples colonias de hormigas es presentado en [25]. El problema de CLRP es descompuesto en dos subproblemas: problema de localización de instalaciones y el problema de ruteo con múltiples depósitos. El algoritmo propuesto utiliza cooperación entre colonias mediante el intercambio de información de feromonas entre la fase de localización y asignación de clientes a depósitos. Este algoritmo es ejecutado 10 veces y los mejores resultados encontrados son reportados con tiempos promedios de cómputo. Para efectos de comparación con los resultados de otros algoritmos para el CLRP, debería considerarse los tiempos completos de cómputo.

La Tabla 3 presenta un resumen de los resultados obtenidos por los algoritmos poblacionales sobre las instancias propuestas.

TABla 3.

RESULTADOS OBTENIDOS PARA ALGORITMOS BASADOS EN POBLACIÓN

\begin{tabular}{|c|c|c|c|c|c|c|c|}
\hline & \multicolumn{2}{|c|}{ Tuzun Instances } & \multicolumn{2}{|c|}{ Prodhon Instances } & \multicolumn{2}{|c|}{ Barreto Instances } & \multirow{2}{*}{ CPU } \\
\hline & Gap BKS & CPU time & Gap BKS & CPU time & Gap BKS & CPU time & \\
\hline MA | PM [23] & 1.86 & 207 & 1.41 & 96 & 2.06 & 36 & $\begin{array}{l}\text { Intel Pentium } 4 \\
\quad(2.40 \mathrm{Ghz})\end{array}$ \\
\hline MACO [25] & 1.23 & 202 & 0.38 & 191 & 0.07 & 49 & $\begin{array}{c}\text { ATHLON XP } \\
2500+(1.83 \mathrm{Ghz})\end{array}$ \\
\hline
\end{tabular}

Fuente: Realizada por los autores.

\section{E. Algoritmos Exactos}

Diferentes formulaciones de dos y tres índices han sido propuestas para el CLRP. Dichos algoritmos han sido capaces de probar optimalidad hasta instancias con menos de 150 clientes. En [26-32] se pueden observar los trabajos representativos en esta categoría. En [26] se presenta el primer algoritmo para resolver de manera exacta el CLRP. La formulación propuesta en este trabajo involucra eliminación de restricciones de subtour. La relajación de la mayoría de las restricciones del problema permite resolver problemas de hasta 20 bodegas potenciales dentro de un número razonable de iteraciones. En Contardo et al. [27-28] se presenta una formulación matemática de dos índices para el CLRP. La solución está basada en generación de columnas, donde los subproblemas consisten en encontrar la ruta más corta de los costos mínimos reducidos bajo restricciones de capacidad. En particular se evalúan todos los subconjuntos disponibles de depósitos que pueden conducir a una solución óptima de costos menores o iguales que un límite superior. Para cada uno de esos subconjuntos, el correspondiente problema de MDVRP es resuelto mediante generación de columnas.
En [29] se propone un algoritmo exacto Branch-andCut para resolver el CLRP. La formulación propuesta está basada en un modelo lineal 0-1 con unas nuevas familias de desigualdades válidas. En [30] se presenta una comparación computacional para cuatro diferentes formulaciones del CLRP. Un algoritmo de Branch-andCut para cada una de las formulaciones es propuesto y comparado en los set de benchmarking para el CLRP. En [31], se describe un nuevo método exacto para resolver el CLRP basado en una set-partitioninglike formulación del problema. Los límites inferiores producidos por diferentes procedimientos, basados en programación dinámica y métodos duales son usados para descomponer el CLRP en un conjunto limitado de problemas de ruteo de vehículos con múltiples depósitos. Finalmente en [32], se presentan algoritmos de aproximación para problemas de optimización para el CLRP.

Cada uno de los algoritmos exactos propuestos se han ejecutado en instancias diferentes a las de benchmarking, lo cual limita la elaboración de una tabla comparativa de resultados. 


\section{F. Algoritmos Combinados}

Los algoritmos combinados, como su nombre lo indica, consideran una mezcla de metodologías de las categorías anteriormente descritas. En [33] se presenta un algoritmo híbrido entre recocido simulado (heurística basada en trayectoria) y colonia de hormigas (heurística basada en población) para el CLRP. En particular, la técnica de recocido simulado es usada para encontrar una buena configuración de centros de distribución, mientras que la metodología de colonia de hormigas es utilizada para encontrar buenas configuraciones de rutas para cada depósito. Las dos metaheurísticas son coordinadas para una exploración eficiente del espacio de solución. En [34], se propone una heurística híbrida basada en esquemas de generación de columnas, donde cada subproblema es resuelto usando un algoritmo de búsqueda tabú. En [35], se propone un algoritmo que toma en consideración las ventajas de las técnicas de simulado y recocido y colonia de hormigas junto con una búsqueda local para resolver el CLRP. Al igual que en [33], el problema de localización se soluciona mediante la técnica de simulado y recocido y el problema de ruteo es abordado por la optimización de colonia de hormigas. En. [36], los autores presentan un algoritmo híbrido similar al propuesto en [33], pero cambiando recocido simulado por búsqueda tabú; siendo esta estrategia la encargada seleccionar los depósitos correctos, y las colonias de hormigas las encargadas de realizar el ruteo en cada uno de los depósitos. En dicho algoritmo no se realizan comparaciones con otros algoritmos efectivos para el CLRP.

En [37], se presenta un algoritmo basado en búsqueda de vecindario variable, usando modelos de programación lineal para explorar cada uno de los vecindarios definidos. Este algoritmo es aplicado a instancias del CLRP, y también en instancias del problema de localización de depósitos y ruteo de vehículos periódico conocido como PLRP (Periodic Location-Routing Problem). En este trabajo, se presenta un algoritmo de solución inicial no tan sofisticado como los propuestos por otros autores, pero según experimentos preliminares, comenzar con un estructura de inicialización simple es bueno para la siguiente fase de optimización (fase de ruteo). En particular, se presentan 5 movimientos, permitiendo encontrar vecinos con cambios en los clientes, como también en los depósitos. También se presentan tres movimientos basados en programación lineal entera para explorar grandes vecindades que permiten reubicar una ruta a otro depósito, o reubicación de clientes individuales entre rutas. El método propuesto logra encontrar buenas soluciones para el CLRP, y es muy destacable en el PLRP, donde logra encontrar mejores soluciones en casi todas las instancias.

En [38] se usa un algoritmo de tipo goloso, el cual se utiliza para generar un conjunto de soluciones iniciales, las cuales se van mejorando por medio de diferentes movimientos de búsqueda local.

En [39], se hace una comparación de diferentes métodos para el CLRP; el primero de ellos es basado en una búsqueda tabú granular publicado en [1], el segundo es un algoritmos de recocido simulado, usando características de búsqueda granular [40,44] y el tercero es una búsqueda tabú granular dentro de una búsqueda de vecindarios variables [21]. En [42], se presenta una búsqueda tabú granular híbrida. Los trabajos citados anteriormente, se componen de dos fases: inicialización, y mejora. En la primera fase se mezclan algoritmos de búsqueda local para problemas del vendedor viajero (TSP), algoritmos de generación de clústeres, modelos matemáticos de programación lineal para selección de depósitos, algoritmos de mejoramiento para el problema de ruteo de vehículos, algoritmos para re-ubicación de clientes, algoritmos de perturbación y algoritmos para eliminar rutas con pocos clientes. En estos trabajos, se destaca lo sofisticado del método de solución inicial, el cual además se encarga de seleccionar los depósitos correctos, ya que en la segunda fase no se realizan movimientos que alteren los depósitos seleccionados; en otras palabras se reduce el espacio de búsqueda. En la segunda fase, se utiliza un espacio de búsqueda granular, con cinco movimientos, guiados por la metaheurística respectiva, además de que se incluyen algoritmos de búsqueda local para el problema de ruteo de vehículos y algoritmos de perturbación para evitar que se pueda obtener un óptimo local fácilmente. Durante la fase de mejora se consideran penalidades dinámicas, las cuales van cambiando de acuerdo a la trayectoria que se sigue, si la búsqueda se mueve por regiones infactibles durante muchas iteraciones, las penalizaciones aumentan; si la búsqueda se mueve por regiones factibles durante muchas iteraciones, las penalizaciones disminuyen.

En [41], se presenta un algoritmo de tres fases, en la primera se genera un conjunto de soluciones factibles 
por medio del uso de GRASP y una búsqueda local. En la segunda fase se utiliza un modelo de programación lineal entero para utilizar las rutas exploradas previamente, recombinarlas, y generar una nueva solución mejorada. Finalmente, en la tercera fase se utiliza una estrategia de generación de columnas para mejorar la solución encontrada en la fase anterior. Esta propuesta encuentra buenas soluciones, mejorando algunas de las mejores soluciones conocidas, pero con tiempo de cómputo elevado.

La Tabla 4 muestra los resultados obtenidos por los algoritmos clasificados dentro de esta categoría.

\section{TABla 4.}

RESULTADOS OBTENIDOS PARA ALGORITMOS COMBINADOS

\begin{tabular}{|c|c|c|c|c|c|c|c|}
\hline & \multicolumn{2}{|c|}{ Tuzun Instances } & \multicolumn{2}{|c|}{ Prodhon Instances } & \multicolumn{2}{|c|}{ Barreto Instances } & \multirow{2}{*}{ CPU } \\
\hline & Gap BKS & CPU time & Gap BKS & CPU time & Gap BKS & CPU time & \\
\hline $\mathrm{TS}+\mathrm{AC}[36]$ & - & - & - & - & 3.20 & - & $\begin{array}{l}\text { Intel Pentium } 4 \\
\quad(2.40 \mathrm{Ghz})\end{array}$ \\
\hline VNS+ILP [37]* & - & - & 0.11 & - & - & - & $\begin{array}{c}\text { Intel Core } 2 \text { Quad } \\
(2.83 \mathrm{Ghz})\end{array}$ \\
\hline ITPH [38] & - & - & 3.58 & - & - & - & $\begin{array}{l}\text { Intel Core i5 } \\
\text { (2.00 Ghz) }\end{array}$ \\
\hline 2 - HGTS [1] & 1.14 & 392 & 0.55 & 176 & 0.78 & 105 & $\begin{array}{l}\text { Intel Core } 2 \text { Duo } \\
\quad(2.00 \mathrm{Ghz})\end{array}$ \\
\hline SA [44] & 1.28 & 368 & - & - & 1.09 & 99 & $\begin{array}{l}\text { Intel Core } 2 \text { Duo } \\
\quad(2.00 \mathrm{Ghz})\end{array}$ \\
\hline $\begin{array}{l}\text { G R A S P + I L P } \\
{[21]^{*}}\end{array}$ & 0.17 & - & 0.11 & - & 0.14 & - & $\begin{array}{c}\text { Intel Xeon E5462 } \\
\text { (3.00 Ghz) }\end{array}$ \\
\hline
\end{tabular}

* Mejores resultados reportados

Fuente: Realizada por los autores

\section{Conclusiones y futuras INVESTIGACIONES}

Para que las compañías mantengan su competitividad logística, es necesario definir metodologías cuantitativas para la estimación de las decisiones estratégicas y operativas en la cadena de abastecimiento. Dos importantes decisiones son la definición de centros de distribución a mantener operando (decisión estratégica) y las rutas para atender a los clientes (decisión operativa). Ambas decisiones se involucran dentro una problemática definida como problema de localización y ruteo con restricciones de capacidad (CLRP). A pesar de que existen diversas metodologías para dar solución al CLRP, no existe una metodología clara y unificada que permita determinar la mejor manera de abordar la problemática en conjunto.
En este paper, nosotros hemos realizado una revisión exhaustiva de la literatura con el fin de dar algunas guías para futuras investigaciones en el área. Con base en la revisión bibliográfica se puede concluir que:

- La mayoría de algoritmos propuestos utilizan set de benchmarking estándar propuestos en la literatura para realizar comparaciones en términos de tiempo y calidad de solución.

- La mayoría de trabajos proponen métodos de solución basados en metaheuristicas, inclusive algunos integrando ideas de métodos de solución exactos (mateheurísticas). Estos algoritmos frecuentemente descomponen el problema en dos fases: localización-asignación (determinar los depósitos a ser a abiertos y los clientes a ser asignados a cada uno de ellos) y ruteo (determinar 
las rutas para atender a los clientes). En algunas ocasiones, las decisiones de asignación son realizadas durante la fase de ruteo. En muchos casos, las dos fases son resueltas iterativamente.

- Los algoritmos heurísticos constructivos han tenido una mayor aplicación práctica como soluciones iniciales para el CLRP. En general dichos algoritmos permiten obtener soluciones iniciales factibles en tiempos de cómputo reducido, las cuales son susceptibles de ser mejoradas mediante algoritmos exactos, algoritmos de trayectoria o algoritmos poblacionales.

- El uso de clústeres de clientes para resolver problemas de ruteo de vehículos con varios depósitos es un área prominente de investigación, debido a que es posible obtener soluciones rápidas que pueden ser mejoradas mediante algún procedimiento de búsqueda local. De esta manera se pueden integrar las metodologías de clúster en un esquema interactivo.

- Heurísticos basados en trayectoria, generalmente han obtenido buenas soluciones dentro de tiempos computacionales reducidos. En particular, estos métodos dividen el CLRP en dos subproblemas: localización y ruteo. Por ejemplo al fijar los depósitos correctos, se puede reducir considerablemente el espacio de solución al pasar de un CLRP a un problema de ruteo de vehículos con múltiples depósitos (MDVRP).

- Varios algoritmos exactos han sido propuestos para el CLRP en los últimos años. Los algoritmos más exitosos han extendido las metodologías propuestas para el VRP al CLRP. A pesar de lo anterior, dichos algoritmos han sido capaces de probar optimalidad en instancias hasta de tamaño mediano (máximo 150 clientes), razón por la cual se ha dado paso a un desarrollo enorme de heurísticas y metaheurísticas para el CLRP.

Nosotros proponemos las siguientes áreas potenciales de investigación:

- Desarrollo de nuevos set de benchmarking: En particular, la mayoría de métodos propuestos para el CLRP se han probado en instancias de hasta 200 clientes y hasta 20 depósitos. Sin embargo, aplicaciones prácticas del CLRP requieren que los algoritmos propuestos sean capaces de obtener soluciones de alta calidad en tiempos de ejecución aceptables para instancias con miles de clientes y cientos de depósitos. Por tal razón el desarrollo de instancias con un tamaño superior a los 200 clientes y 20 depósitos podría ser útil.

- Desarrollo de metaheurísticas unificadas: El desarrollo de metodologías unificadas y robustas permitirá resolver la problemática de manera adecuada, evitando la proliferación de algoritmos metaheurísticos con características similares. Los algoritmos diseñados debería ser eficientes para la solución de instancias de gran tamaño.

- Estrategias de paralelismo: Dichas estrategias podrían permitir una mejor exploración del espacio de solución dentro de tiempos computacionales razonables. En particular, se podría extender algoritmos metaheurísticos paralelos propuestos para diversas variantes del VRP ([45-49]).

- Desarrollo de metodologías sistemáticas para el diseño de experimentos: El desarrollo de metodologías unificadas para realizar la calibración de parámetros, es un elemento fundamental para la comparación de las metodologías propuestas.

- Diseños de nuevos algoritmos exactos: Desarrollo de metodologías exactas que permitan explotar más la estructura del problema.

- Análisis de eficiencia de las metaheurísticas propuestas: En la actualidad no existe estudio alguno que identifique con detalle las fortalezas y el grado de sofisticación de un algoritmo frente a otro. De igual manera, el contraste de los algoritmos exactos y las metaheurísticas es una discusión muy valiosa en términos de la eficiencia en la solución del CLRP.

\section{REFERENCIAS}

[1] J. W. Escobar, R. Linfati and P. Toth, "A two-phase hybrid heuristic algorithm for the capacitated location-routing problem", Computers \& Operations Research,vol. 40 (1), pp. 70-79, 2012. 
[2] G. Rand, "Methodological choices in depot location studies",Operational Research Quarterly, vol. 27 (1), pp. 241-249, 1976.

[3] D. Tuzun, and L.I. Burke, "A two-phase tabú search approach to the location routing problem", European Journal of Operational Research, vol. 116(1), pp. 87-99, 1999.

[4] C. Prins, C. Prodhon and R.W. Calvo."Solving the capacitated location-routing problem by a GRASP complemented by a learning process and a path relinking", 4OR: A Quarterly Journal of Operations Research, vol. 4(3), pp. 221-238, 2006.

[5] S. Barreto et al., "Using clustering analysis in a capacitated location-routing problem", European Journal of Operational Research, vol. 179(3), pp. 968-977, 2007.

[6] H. Min, V. Jayaraman and R. Srivastava "Combined location-routing problems: A synthesis and future research directions", European Journal of Operational Research, vol. 108 (1), pp. 1-15, 1998.

[7] G. Nagy and S. Salhi, "Location-routing: issues, models and methods",European Journal of Operational Research, vol. 177 (2), pp. 649-672, 2007.

[8] A. Hassanzadeh et al., Location-Routing Problem. Facility Location: Springer Verlag, 2009.

[9] C. Prodhon and C. Prins, "A survey of recent research on location-routing problems", European Journal of Operational Research, vol. 238(1), pp. 1-17, 2014.

[10] C. Duhamel et al., "A GRASP $\times$ ELS approach for the capacitated location-routing problem", Computers \& Operations Research, vol. 37(11), pp. 1912-1923, 2010.

[11] G. Clarke and J.W. Wright, "Scheduling of vehicles from a central depot to a number of delivery points", Operation Research,vol.12, pp. 568-581, 1964.

[12] S. Wolf and P. Merz, "Evolutionary local search for the super-peer selection problem and the p-hub median problem", Lecture Notes in Computer Science, vol. 4771, pp. 1-15, 2007.

[13] R. B. Lopes et al.,"A decision-support tool for a capacitated location-routing problem", Decision Support Systems, vol. 46(1), pp. 366-375, 2008.

[14] A. Nadizadehet al., "Using greedy clustering method to solve capacitated location-routing problem", African Journal of Business Management, vol. 5(21), pp. 8470-8477, 2011.

[15] C. Prins et al., "Solving the capacitated locationrouting problem by a cooperative Lagrangean relaxation-granular tabú search heuristic", Transportation Science, vol. 41(4), pp. 470-483, 2007.

[16] V. F. Yu et al., "A simulated annealing heuristic for the capacitated location routing problem",Computers \& Industrial Engineering, vol. 58(2), pp. 288-299, 2010.

[17] M. S. Jabal-Ameli, M.B. Aryanezhad and N. Ghaffari-Nasab, "A variable neighborhood descent based heuristic to solve the capacitated location-routing problem", International Journal of Industrial Engineering Computations, vol. 2(1), pp. 141-154, 2011.

[18] H. Derbel et al., "A variable neighborhood search for the capacitated location-routing problem", In 4th International Conference on Logistics (LOGISTIQUA), pp. 514-519, IEEE, 2011.

[19] V. C. Hemmelmayr, J. F. Cordeau and T. G. Crainic, "An adaptive large neighborhood search heuristic for two-echelon vehicle routing problems arising in city logistics",Computers \& Operations Research, vol. 39(12), pp. 3215$3228,2012$.

[20] A. Jokar and R. Sahraeian, "A Heuristic Based Approach to Solve a Capacitated Locationrouting Problem", Journal of Management and Sustainability, vol. 2(2), pp. 219-226, 2012.

[21] J. W. Escobar et al., "A Granular Variable Tabú Neighborhood Search for the capacitated location-routing problem", Transportation Research, Part B: Methodological, vol. 67, pp. 344-356, 2014.

[22] S. Ropke and D. Pisinger, "An adaptive large neighborhood search heuristic for the pickup and delivery problem with time windows",Transportation Science,vol. 40, pp. 455-472, 2006.

[23] C.Prins, C.Prodhon and R.W.Calvo, "AMemetic Algorithm with Population Management (MA|PM) for the Capacitated Location-Routing Problem", Lecture Notes in Computer Science, vol. 3906, pp. 183-194, 2006.

[24] C. Duhamel et al., "A memetic approach for the capacitated location routing problem", In Proceedings of the 9th EU/Meeting on 
Metaheuristics for Logistics and Vehicle Routing, Troyes, France. 2008.

[25] C. J. Ting and C. H. Chen, "A multiple ant colony optimization algorithm for the capacitated location routing problem", International Journal of Production Economics, vol. 141(1), pp. 3444, 2013.

[26] G. Laporte, Y. Nobert and D. Arpin, "An exact algorithm for solving a capacitated locationrouting problem", Annals of Operations Research, vol. 6(9), pp. 291-310, 1986.

[27] C. Contardo, J. F. Cordeau and B. Gendron, "A branch-and-cut algorithm for the capacitated location-routing problem", In Conference CAOR 10, 2010.

[28] C. Contardo, B. Gendron and J. F. Cordeau, A branch-and-cut-and-price algorithm for the capacitated location-routing problem, CIRRELT, 2011.

[29] J. M. Belenguer et al., "A branch-and-cut method for the capacitated location-routing problem", Computers \& Operations Research, vol. 38(6), pp. 931-941, 2011.

[30] C. Contardo, B. Gendron and J. F. Cordeau, A computational comparison of flow formulations for the capacitated location-routing problem, CIRRELT, 2011.

[31] R. Baldacci, A. Mingozzi and R. W. Calvo, "An exact method for the capacitated location-routing problem", Operations Research, vol. 59(5), pp. 1284-1296, 2011.

[32] T. Harks, F. G. Konig and J. Matuschke, "Approximation Algorithms for Capacitated Location Routing", Technische Universitat Berlin, Institut fur Mathematik Straße des 17.Germany, 2012.

[33] L. Bouhafs and A. Koukam, "A combination of simulated annealing and ant colony system for the capacitated location-routing problem", In Knowledge-Based Intelligent Information and Engineering Systems, pp. 409-416, Springer Berlin Heidelberg, 2006.

[34] C. Boulanger and F. Semet, "A column generation based heuristic for the capacitated location-routing problem", In EU/Meeting Conference, EURO 2008.

[35] J. Yan et al., "A hybrid ant colony algorithm for the capacitated location-routing problem",3rd International Conference on Intelligent System and Knowledge Engineering, pp. 38-43. IEEE, 2008.

[36] L. Bouhafs, A. Hajjam and A. Koukam, "A Tabú Search and Ant Colony System Approach for the Capacitated Location-Routing Problem".Ninth ACIS International Conference on Software Engineering, Artificial Intelligence, Networking, and Parallel/Distributed Computing, pp. 46-50, IEEE, 2008.

[37] S. Pirkwieser and G. R. Raidl, "Variable neighborhood search coupled with ILP-based very large neighborhood searches for the (periodic) location-routing problem", In Hybrid Metaheuristics, pp. 174-189, Springer Berlin Heidelberg, 2010.

[38] A. Jokar and R. Sahraeian, "An Iterative two Phase Search Based Heuristic to Solve the Capacitated Location-Routing Problem", Australian Journal of Basic \& Applied Sciences, vol. 5(12), pp. 1613-1621, 2011.

[39] J. W. Escobar et al., "A comparison of three etaheuristic algorithms for the capacitated location-routing problem",Proceedings of the fifth International Workshop on Freight Transportation and Logistics (ODYSSEUS), pp. 560-563, 2012.

[40] J.W.Escobar etal., "Acomputational comparison of heuristic algorithms for the capacitated location-routing problem", Technical Report, University of Bologna, 2012.

[41] C. Contardo, J. F. Cordeau and B.Gendron, "A GRASP + ILP-based metaheuristic for the capacitated location-routing problem",Technical Report CIRRELT, 2011.

[42] J. W. Escobar, R. Linfati and P. Toth, "A hybrid metaheuristic algorithm for the capacitatedlocation routing problem", Proceedings of CLAIO/SBPO, Rio de Janeiro-Brazil, September 24-28, 2012.

[43] J. W. Escobar et al., "A metaheuristic algorithm based on a Granular Tabú Search within a Variable Neighborhood Search for the capacitated location-routing problem", Proceedings of VEROLOG - First meeting of the EURO Working Group on Vehicle Routing and Logistics Optimization, pp. 78-79, BolognaItaly, June 18-20, 2012.

[44] J. W. Escobar and R. Linfati, "Un algoritmo metaheurístico basado en recocido simulado con espacio de búsqueda granular para el problema 
de localización y ruteo con restricciones de capacidad", Revista Ingenierías Universidad de Medellín, vol. 11(21), pp. 139-150, 2012.

[45] J. Jin, T. G. Crainic and A. Løkketangen, “A parallel multi-neighborhood cooperative tabú search for capacitated vehicle routing problems", European Journal of Operational Research, vol. 222(3), pp. 441-451, 2012.

[46] P. Badeau et al., "A parallel tabú search heuristic for the vehicle routing problem with time windows", Transportation Research, Part C: Emerging Technologies, vol. 5(2), pp. 109-122, 1997.
[47] J. F. Cordeau and M. Maischberger, "A parallel iterated tabú search heuristic for vehicle routing problems", Computers \& Operations Research, vol. 39(9), pp. 2033-2050, 2012.

[48] L. S. Ochi et al., "A parallel evolutionary algorithm for the vehicle routing problem with heterogeneous fleet. In Parallel and Distributed Processing, Springer Berlin Heidelberg, pp. 216224,1998.

[49] A. Subramanian et al., "A parallel heuristic for the vehicle routing problem with simultaneous pickup and delivery", Computers \& Operations Research, vol. 37(11), 1899-1911, 2010. 